\title{
Transforming creativity: Personalized manufacturing meets embodied computing
}

\author{
Robert E. McGrath* \\ Champaign Urbana Community Fab Lab \\ Art Annex 2 \\ 1301 South Goodwin Avenue, Urbana, Illinois 61801, USA \\ E-mail: Robert.mcgrath216@gmail.com
}

\section{Johann Rischau}

The Product Manufactory

722 Killarney Street, Urbana, Illinois 61801, USA

E-mail: johann@tpm-team.com

\author{
Alan B. Craig \\ Institute for Computing in Humanities, Arts, and Social Science \\ National Center for Supercomputing Applications \\ University of Illinois, Urbana-Champaign, USA \\ E-mail: acraig@ncsa.illinois.edu \\ *Corresponding author
}

\begin{abstract}
This paper discusses aspects of a collaborative investigation of embodied computing and personal manufacturing. We describe the NeuroMaker 1.0, an artwork that playfully implements the concept of "translating of the designer's ideas into a product". Visitors to the installation were invited to use their own EEG to fabricate personalized physical objects. While primarily intended to provoke thought about the process of creativity, we also demonstrated that, with the right team, radical new interfaces are well within the reach.
\end{abstract}

Keywords: Personal fabrication; Embodied computing; Creativity; Transforming

Biographical notes: Robert E. McGrath is retired from the National Center for Supercomputing Applications at the University of Illinois, Urbana-Champaign. $\mathrm{He}$ has been a software engineer for 30 years, in academic and commercial projects, and has contributed to the development of high performance computing, the World Wide Web, digital libraries, and novel human computer interfaces.

Johann Rishau completed is MFA in the department of Art and Design at the University of Illinois, Urbana Champaign in 2011. He is co-founder of The Product Manufactory, a custom product development company in Urbana, Illinois. 
Alan B. Craig is Senior Associate Director for Human-Computer Interaction of the Institute for Computing in Humanities, Arts, and Social Science (I-CHASS) at the University of Illinois at Urbana-Champaign. He has pioneered development of Virtual Reality, as well as the application of high performance computing and advanced interfaces to science, education, and the humanities.

\section{Introduction}

For more than a century, goods have been mass-produced through a set of specialized roles of consumer, designer, distributor, and manufacturer. In this arrangement, industrial design processes do not necessarily accommodate the possibility of the user altering the design, other than a few predetermined choices of color or patterns (Pine, 1993; Rischau, 2011). Personal fabrication technology coupled with on-line collaboration and open distribution of designs challenges these practices, and enables not only "customization", but also "mashing up" designs, as well as original creation. These technologies make possible, and call for, collaborative design: systems must enable designers and users to jointly create products. A designer who attempts to create the one optimal object may not satisfy as many people as the designer who creates a good toolkit, with open designs that are easy to modify, adapt, or coopt.

If the end-user is to directly participate in product design, there must be accessible interfaces through which to control sophisticated design tools and fabrication processes. Today, personal fabrication requires significant personal investment to learn both the tools and how to use them. While the cost of equipment has dropped significantly, and design tools are readily available, it is still necessary to learn about materials, design, and fabrication processes. And most importantly, simple, easy to use, yet expressive, interfaces are needed, to enable non-experts to control the digital design process.

At the same time, digital systems make it possible to create an unlimited variety of virtual worlds, which exist in parallel with physical reality (Castranova, 2007; Krueger, 1991; Laurel, 1991; Sherman \& Craig, 2003). Humans can interact with these digital systems in a variety of modes, from mouse-keyboard-screen through speech, vision, and motion. These interactive systems can sense the physical world, and can manipulate aspects of the physical world, creating experiences that blur the boundaries of the physical and virtual, crossing and linking between real and virtual.

These digital technologies create not just a new medium, but a whole universe of new media. The new media encompass a broad space of "mixed reality", using different combinations of sensors and displays to create digitally enhanced experiences. Broadly speaking, these include immersive video games (e.g., Castranova, 2007; Williams et al., 2006), Alternate Reality Games played on city streets (McGonigal, 2006), Virtual Reality in which digital displays take over many senses (Sherman \& Craig, 2003), and Augmented Reality which inserts digital content into the physical world (e.g., Billinghurst, Kato, \& Poupyrev, 2001; McGrath, Craig, Bock, \& Rocha, 2011).

The development of low cost sensors has opened the way to radical transformation of human computer interfaces, incorporating the entire body (England, 2011a; Hornecker, 2011; Isbister, 2011; Ishii \& Ullmer, 1997; Moss, 2011; Pentland, 2008; Pietrowicz, McGrath, Garnett, \& Toenjes, 2010; T. Schiphorst, 2009a; Smith \& Garnett, 2011b; Van Laerhoven \& Cakmacki, 2000). Real time sensing opens the way for 
interfaces in which people may communicate with their computer by gesturing, talking, singing, and dancing.

In this paper we discuss one exploration that integrates concrete examples from this space: embodied computing and personal fabrication. Digital fabrication technology has enabled the rise of personal fabrication, which places the means of production in the hands of the consumer. As discussed below, product designs are created in a virtual world, and then fabricated by digitally controlled machines. This technology not only transforms manufacturing, it enables new forms of sharing and collective creativity, and sets the stage for a transformation of economics, and a redistribution of the creative roles (Gershenfeld, 2005; Mota, 2011; Rischau, 2011).

At the same time, the widespread availability of sensors, networks, and powerful computers makes possible new types of interactions between humans and computers and between humans mediated by computers. Embodied computing, or Whole Body Interfaces, in which a human can interact with the virtual world using his or her whole body, promise to radically transform everyday life and potentially engender new forms of creative engagement (England, 2011a; Pietrowicz et al., 2010; Schiphorst, 2009a).

Each of these technologies is transformative alone, and because they are digitally mediated, we can combine them relatively easily to create another experience altogether: combining these technologies defines a computer-mediated creative environment in which a person can express a design with his or her whole body, and then create a physical object that realizes that design. This vision suggests a radical new mode of creative work, not only "personalized" in the sense of "consumer selected", but also personally expressed through the individual's own gesture, sound, and thought. The work discussed here was inspired by thought experiments, in which we sought to envision what might be possible in such an environment. For example, imagine that one might "sculpt" the design of a chair by dancing, then "print" the result in plastic or wood.

We have illustrated these concepts in an art installation, called "the NeuroMaker 1.0" (McGrath, Rischau, \& Craig, 2011; NCSA, 2011). The NeuroMaker was realized as an artwork that combined aspects of embodied computing and personal fabrication. During its exhibition, visitors were able to use their own brainwaves to create customized designs, and then realize the design using a digitally controlled fabrication machine.

As an artwork, the NeuroMaker was not intended to demonstrate a practical technology. Rather, it takes literally the abstract notion of "translating the thought of a designer into a product", and, by attempting to create a system that does exactly that, perhaps reveal some of the essence of the design and fabrication process. In addition, we tested what could be achieved today within a limited budget.

This project emerged from a series of collaborations made possible by an institutional context, including the Champaign Urbana Community Fab Lab (Watson, 2011) and several virtual institutes at the University of Illinois, Urbana-Champaign (eDream, 2011; I-CHASS, 2009; IACAT, 2011; Illinois Informatics Institute, 2012). These institutions bring together technicians, artists, teachers, and students from many backgrounds, to bring to bear diverse theories and practices, through mutual teaching, learning, and translation across conceptual boundaries.

This paper is organized as follows. Section 2 reviews the technologies and previous work. Section 3 describes the NeuroMaker prototype. Section 4 discusses the implications of this project and concludes. 


\section{Background}

The NeuroMaker project came together during discussions of how to apply novel human computer interfaces to take a fresh look at design methodologies and tools for personal fabrication. In this project, we called upon emerging techniques in embodied computing, applied to the task of personalizing the fabrication of objects. This section discusses some of the foundations of our work, briefly defining and reviewing personal fabrication and embodied computing.

\subsection{Design and fabrication}

Personal fabrication has become possible through the rise of digital fabrication technology. "Digital Fabrication" refers to digitally augmented design and fabrication processes. Designs are created with CAD/CAM software that enables one or more person to visualize and precisely specify three-dimensional objects. The designs may be drawn by hand, created by computer simulation, may incorporate data from two- and threedimensional imagery, or may use combinations of all these sources.

The resulting designs are represented as digital data that can be processed, shared, or stored for later use. The digital data also can be algorithmically manipulated in a variety of modes, including:

- Simulation and analytic analysis (e.g., to model the properties of materials and structures),

- Application of heuristic knowledge (e.g., recommended limits for human comfort), and

- Parameterized and generative processes (e.g., to instantiate components from a family of related designs).

When a design is complete, the data that describes it can be sent to a variety of computer-controlled machines, which may use subtractive or additive methods to create objects from a variety of materials. The digital data not only enables extremely precise and replicable results, it opens the way to reuse, sharing, and even new forms of "publication" of designs, e.g., by sharing files over the Internet.

Developed for mass production and precision engineering, these technologies have evolved to the point that the entire workflow is now also available in inexpensive, open technology potentially available to anyone--"personal fabrication". The rise of personal fabrication has begun the democratization of design and manufacturing in new businesses, at home, and in small, local organizations. Thousands of non-professionals are now engaged in design, adaptation, and fabrication of objects, which has emerged in many forms of "do it yourself" (DIY) culture, variously known as "makers" (Make, 2011), Fab Labs (Gershenfeld, 2005), Open Source Hardware (LilyPond, 2011; Makerbot Industries, 2011a, 2011b; openhardwaresummit.org, 2011; Wohlsen, 2011) and a vibrant Internet culture (Fluidforms, 2011; Makerbot Industries, 2011b; New Media Collective, 2011; shapeways.com, 2011). This technology-driven culture has produced a burst of creativity, characterized by the sharing of design and knowhow, new modes for fabrication and distribution, and new digital services.

These developments in digital technology and culture are transforming the creation of physical objects, with profound implications for future of industrial design, manufacturing, and creativity (Mota, 2011; Rischau, 2011). Among other transformations, it is becoming possible to not only manufacture on demand, but also to fabricate at a location of choice, be it a factory, retail store, or home. Also, the emergence of a "maker" culture increases demand for personalized products, and for designs that allow 
customization. This is an opportunity for designers to attract customers by providing opportunities for personalized creation of products. Customers may be engaged through well-designed computer interfaces with corresponding underlying processes, that encourage customers to participate in the design of the product (Rischau, 2011).

\subsection{Embodied computing}

For several decades, humans have generally interacted with computers through keyboards, screens, and pointing devices. These interactions use a very limited extent of human capabilities, mostly hand and finger movements, with visual and audio presentations (England, 2011a). In contrast, everyday interaction with the world uses many modalities, including production of sound (speech, vocalization, music), physical gesture (movement of body, head, eyes, object manipulation), and all-around vision (image, text, lighting) (Pietrowicz et al., 2010).

Recent developments in low cost sensors have opened the way to human computer interfaces that incorporate the entire body (England, 2011a; Hornecker, 2011; Isbister, 2011; Ishii \& Ullmer, 1997; Moss, 2011; Pentland, 2008; Pietrowicz et al., 2010; T. Schiphorst, 2009a; Smith \& Garnett, 2011b; Van Laerhoven \& Cakmacki, 2000). Computer interfaces can use data from digital audio, video, and sensors that measure of pressure, acceleration, and other physical phenomena. Sensors may track both "overt" data such as movement and facial expression, and "covert" data such as breathing, heartbeat, and brain activity.

This technology requires real time data analysis to extract and track multiple features at many temporal resolutions to create a rich stream of signals that represents behavior of the person. These signals are computed from sensed speech, motion, gesture, and physiological phenomena. These digital signals can be combined and analysed to discover meaningful patterns, at various levels of abstraction. Current research is exploring techniques for recognizing meaningful patterns in such data, and also developing conceptual models of what patterns might be meaningful and how to integrate multiple signals (Pentland, 2008; Pietrowicz et al., 2010; Smith \& Garnett, 2011a, 2011b; Van Laerhoven \& Cakmacki, 2000).

There are two important aspects of the analysis process: feature extraction and semantic mapping. A whole body interface will extract many features from what it senses about the person's body; then the features are then given meaning by the system. Feature extraction is the process of discovering meaningful signals in the raw data. For example, a WiiMote (Nintendo, 2010; Wikipedia, 2011d) may tell the computer where it is and what direction it is moving, but the computer needs to use that information to discover patterns that characterize gestures and other meaningful movements.

The relationship between features and meaningful concepts is a semantic mapping. For example, the computer may detect the person is hopping up and down, and this may be declared to mean, "I'm ready to start." One approach uses streams of sensor data to generate abstract multimodal "gestures" (Mistry, Maes, \& Chang, 2009; Pietrowicz et al., 2010; Smith \& Garnett, 2011a, 2011b), which can operate as input for computer systems. In this way, a computer program might be controlled by a combination hand and body movements (even through dance), sounds (including music), or physiology (such as heartbeat or brain activity). 


\section{The NeuroMaker 1.0}

In this paper, we discuss one prototype, the "NeuroMaker 1.0", that was built and exhibited as part of an installation in February 2011 (McGrath, Rischau, \& Craig, 2011; NCSA, 2011). The NeuroMaker was developed as part of an examination of the future of mass customization made possible by technologies that allow us to create objects based on digital data that can be manipulated to change the outcome. At the same time, this work also explored a simple example of how "whole body interfaces" may enable more direct interaction with the physical world, with the computer playing a less intrusive role.

Design and fabrication can be abstractly described as a process whereby the thoughts of the designer is transmitted into physical objects. Design and fabrication tools and their human interfaces mediate this process. The ultimate user's thoughts and actions are represented via the designer's mental models of the consumers, which drive the realization of one or more products. The goal of mass customization is to allow the user to also play the role of designer, and also to create a shorter, more direct path from thought to product.

The NeuroMaker was born from the playful attempt to take this abstract picture literally; to imagine a system in which thoughts are directly translated to designs and products. The art installation helped bring these ideas into clearer view, and gives an opportunity for critical thought about this view of the design process. To the degree that the NeuroMaker falls short, it also illustrates open questions about the potential value and use of such a direct interface.

The project began with playful musings, such as:

- How can we as humans emotionally connect to objects?

- How can Industrial Designers use the technology of digital fabrication to create product concepts that embody a part of the end consumer personality?

- How can a consumer directly participate in the fabrication of an object, without the mediation of a designer?

- How should computer interfaces be designed to enable more direct creation of objects?

From these discussions, we sought to create a working system through which a personal object can be created, based directly on the actions and thoughts of the individual person at this moment in time. The result was the "NeuroMaker": a personal fabrication system that can be controlled through a person's live Electroencephalogram (EEG). The measurements of brain activity are analysed and used to create a customized decorative pattern that is drawn or etched onto an object as it is manufactured.

The NeuroMaker was designed as a demonstration as part of an art installation, and as such, it was primarily intended to stimulate imaginative thought about creativity, personal fabrication, and embodied computing. The work did not pretend to be a complete solution or usable product, and we specifically do not claim to have made scientifically valid measurements of human thought or emotions.

The NeuroMaker enables a person to operate a personal manufacturing system with minimal technical knowledge, to create objects that "represent" their internal thoughts. An example product could be a "Mindmemo": the system makes an "image" of your unique thoughts and lets you "put them into a box". The design template is a foldup, tabbed box that is cut out and scored by a routing machine. A pattern derived from your brain waves would be inscribed (e.g., drawn in ink or etched into the surface) on the inside surface of the box. The result is a box "containing" a personal thought, a thought 
you make keep private or "open up" to show to others as you choose. Figure 1 illustrates the overall process.

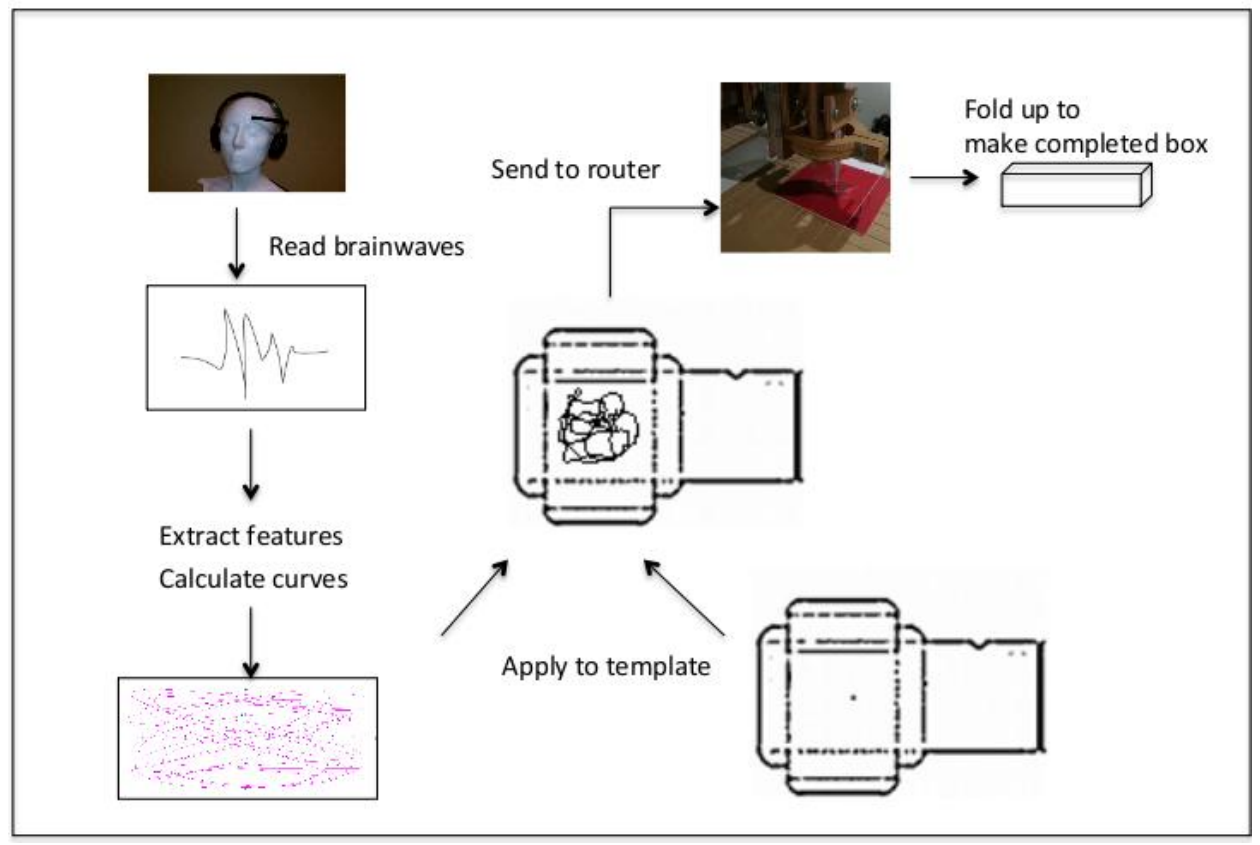

Fig. 1. Example workflow for NeuroMaker

\subsection{Technical description}

The NeuroMaker is a combination of three different elements: a computer controlled router (for cutting parts to build objects), an inexpensive EEG sensor (for human interaction), and data analysis software to algorithmically map sensor input to design elements in the manufactured object. The NeuroMaker integrates these relatively inexpensive and readily available components to create a unique interactive computer experience.

\subsubsection{Computer-aided fabrication}

The CNC Router was built from a kit (available from (buildyourcnc, 2011)). The completed router is controlled by the Mach3 software application that executes designs stored in computer files (ArtSoft, 2011). The computerized router is capable of precisely reproducing the same cuts every time it executes-i.e., it can mass-produce a digital model. One or more people create a digital design using software tools. The resulting design is stored as data for the CNC Router.

Operating the CNC Router requires some expertise, though the NeuroMaker automated the process to be simple enough to learn in a few minutes. Operating power machinery entails some danger, noise, and dust, and should not be done carelessly. For this reason, in the public art installation, a knowledgeable operator executed the manufacturing step, using the patrons' designs. 


\subsubsection{Sensing the user's body}

The NeuroMaker uses an EEG device to sense the "mental state" of the user. By attaching electrodes to the scalp and face, it is possible to record electrical activity in the human brain. The "brainwaves" that are detected, termed electroencephalography (EEG), may reveal the functioning of the brain.

Hospitals and scientific laboratories have studied electroencephalography for nearly a century (Niedermeyer \& da Silva, 1982; Swartza \& Goldensohnb, 1998; Wikipedia, 2011a); it is now possible to build inexpensive and simple-to-use EEG sensors, which can be connected to any computer. These, and other "Brain Computer Interfaces" enable the computer to sense a person's brainwaves, and, consequently, to let the person control the computer just by thinking (McFarland \& Wolpaw, 2011; Vallabhaneni, Wang, \& He, 2005).

The NeuroMaker uses one such sensor, the Mindset, from NeuroSky (NeuroSky, 2011). This device connects to a PC or laptop via Bluetooth. The Mindset measures electrical activity via electrodes on the ear and forehead, and sends data to a computer. The data represents the raw EEG trace, based on measured voltages in the brain, sampled approximately 500 times per second. The NeuroSky software also reports eight commonly recognized frequency bands of EEG signals (NeuroSky, 2010a). The data from the sensor can be read using commonly available software, such as software from JSON.org (Json.org, 2011). For details of the data format, please see (NeuroSky, 2010b).

\subsubsection{Feature extraction and mapping}

Abstractly, the NeuroMaker software operates by sampling the EEG signal (i.e., the sensor data) over one or more time periods, and extracting (i.e., computing) one or more features to represent the data. The details of the sampling and feature extraction are programmable; the NeuroMaker software could implement many possible alternatives.

Obviously, no handful of numbers can be a perfect picture of a human brain, so the challenge is to find features that give good enough information to be useful for a given purpose. In the case of the NeuroMaker, we used several variants of a very simple sampling technique. This was not a scientific study, so this approach served well enough to demonstrate the principle in the context of the art installation.

The extracted features are "mapped" to decorative curves or patterns by applying them as parameters to a pair of generative functions. The NeuroMaker uses software adapted from David Eck's "Java Components for Mathematics" (Eck, 2001) to compute parametric curves and display them on the computer screen. The equations used in art installation were:

$$
\begin{aligned}
& \mathrm{x}_{\mathrm{t}}=\mathrm{x}_{1} \cdot \sin \left(\mathrm{x}_{2} \cdot \mathrm{t}\right)+\sin \left(\mathrm{x}_{3} \cdot \mathrm{t}\right) \\
& \mathrm{y}_{\mathrm{t}}=\mathrm{x}_{4} \cdot \cos \left(\mathrm{x}_{5} \cdot \mathrm{t}\right)+\sin \left(\mathrm{x}_{6} \cdot \mathrm{t}\right)
\end{aligned}
$$

where $\mathrm{x}_{\mathrm{i}}$ are six values extracted (sample) from the input data, as described above.

The functions are used to construct a winding curve, by setting $t$ to 0 through 1,000 to obtain 1,000 ordered pairs, $\left(x_{t}, y_{t}\right)$. The resulting curves are very sensitive to the input data, such that very tiny differences in the six input values yields visibly very different curves. For this reason, it would be very unusual to ever see a duplicate curve.

The NeuroMaker renders these designs using two styles of drawing, both based on the same parametric curves. One style is a continuous, winding curve reminiscent of a Celtic knot. The other style is a series of rectangles, plotted along the path of the curve, 
proportional to the curvature. Figure 2 illustrates example drawings of the two styles, rendered from the same input parameters.

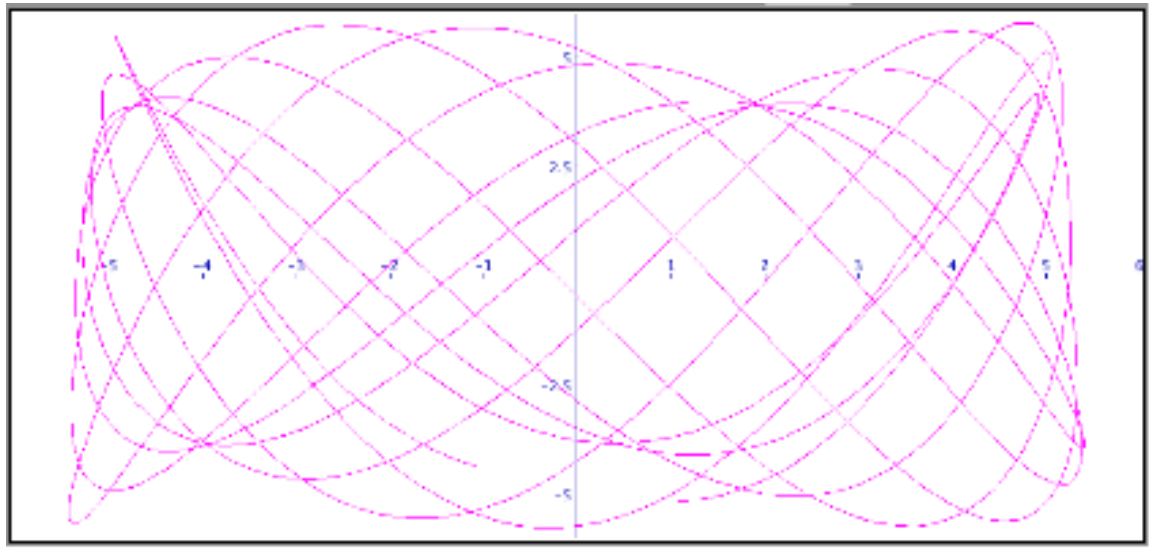

(a)

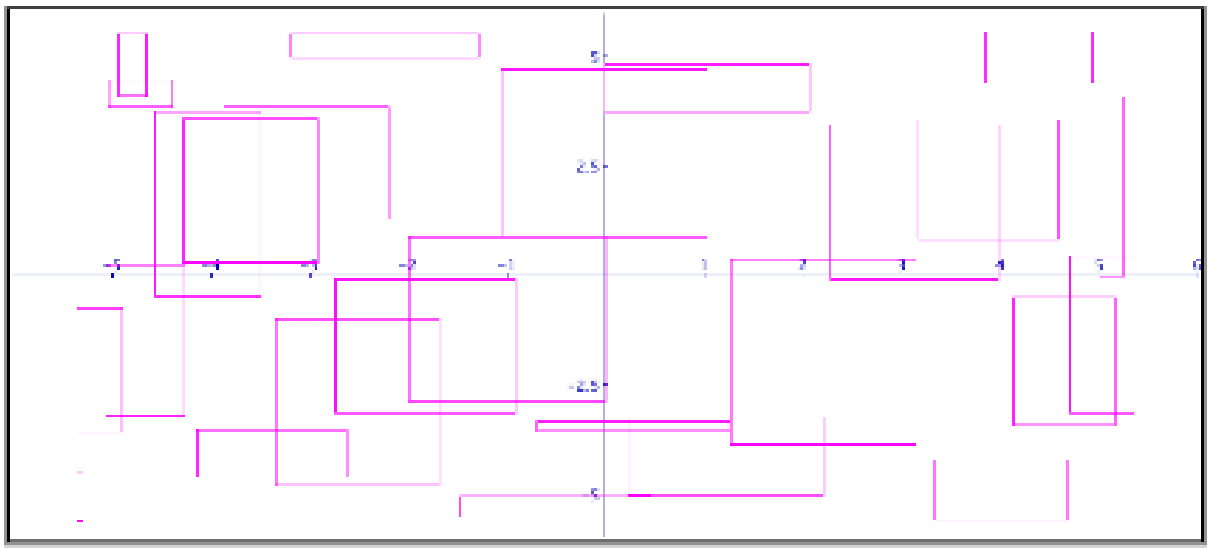

(b)

Fig. 2. Example of two alternative decorative patterns rendered from the same parameters. The calculated curve is rendered in different styles, such as a continuous curve (a) or sequence of rectangles (b)

When the user is satisfied with the decorative pattern, it is merged with a design template to create a unique, customized data file for his own product. For example, the decorative pattern would be applied to the template, so that the machine will be instructed to inscribe it on the surface of a cut out box. Technically, this is realized by converting the parametric curves into G-code (Smid, 2008; Wikipedia, 2011b) which is merged with template G-code describing the object. The customized data file is then "printed" to the CNC Router, using Mach3 software from ArtSoft (ArtSoft, 2011), to create a personalized version of the article. 


\subsection{Limitations of the NeuroMaker prototype and installation}

While the specific device created was not intended to be a complete solution, and the art installation was not intended as a scientific study, it is important to note several of the important limitations of the prototype described above.

\subsubsection{Sensing and feature analysis}

In general, EEG data represents unlocalized activity of the whole brain. This type of aggregate data has never been shown to reflect thoughts or emotions except in a very broad sense, and it is not likely that any features extracted from the data would have much valid relationship to thoughts or emotions of the user. The data produced by the NeuroSky sensor is, in this sense, too coarse, and not likely to be useful for detailed analysis of "mental state".

More sophisticated (and much more costly) sensors (e.g., some form of Magnetic Resonance Imaging (MRI) (Haacke, Brown, Thompson, \& Venkatesan, 1999; Wikipedia, 2011c)) could provide much better data, including measures of localized brain functions. Improved sensing would make it possible to develop much more sophisticated feature analysis. However, even if an inexpensive and portable MRI device were available, developing effective feature analysis for the data would require significant investigation and trials, far beyond the scope of this project.

With these limitations in mind, the prototype did not attempt to extract a "meaningful" representation of the brain activity. Instead, we relied on a simple sampling algorithm. Whether the samples had any relationship to the person's mental state is highly debatable, but the system did produce pleasing results.

The NeuroMaker software is designed to allow the use of alternative feature analysis methods, which could be based on a variety of machine learning techniques emerging from resent research (e.g., (Pentland, 2008; Van Laerhoven \& Cakmacki, 2000; Wang, 2011)). Such techniques are the subject of current research (Pietrowicz et al., 2010; Smith \& Garnett, 2011b), but were not yet mature enough to be used in the NeuroMaker.

The prototype used one type of sensor, an EEG, though there are many potential sensor sources, including gestures, sound, and movement (e.g., Latulipe et al., 2010; Mistry et al., 2009; Pietrowicz et al., 2010; Smith \& Garnett, 2011b). Ongoing research is developing techniques to combine multiple sensors would be combined into a "meta" control, which might be used to in a system like the NeuroMaker.

\subsubsection{The template and customization process}

The NeuroMaker used a very simple form of customization, in the form of a template object with specific surfaces reserved for customized designs. The decorative pattern was generated using a parameterized system of equations, but only a limited area of the whole object was parameterized in this way.

Thus, in this project we employed very simple forms of two important design patterns for mass customization: interchangeable "plug in", and parameterized algorithm. Other approaches to customization might be considered, including direct manipulation (e.g., Ishii \& Ullmer, 1997), constructive design (e.g., Xiu, Wan, \& Cao, 2011), or learning by example (e.g., Norton, Heath, \& Ventura, 2011). 


\subsubsection{The fabrication system}

The NeuroMaker used a specific CNC milling machine, which has its limitations. The specific machine used was capable of cutting a range of materials (cardboard, wood, foam), with moderate precision, and individual parts limited to approximately 12 " x 24 " $\mathrm{x}$ 4". There are many other machines that might be used, which could create a wider variety of objects using other materials (such as metals or ceramics), with much larger form factors, and higher precision. Since the development of the template object involved experiments and tuning with the $\mathrm{CNC}$ router itself, using another device would probably require additional testing and adjustment to the templates.

In general, fabrication machines are too dangerous and expensive for unsupervised operation by untrained individuals. For the public installation, the fabrication step employed the service of a trained operator who loaded the material, readied the system, loaded each person's design, and maintained safe operation while the object was fabricated. However, most people could learn to run the machine in about an hour.

\section{Summary and discussion}

Our work is exploring the boundaries of the virtual and physical, where digital audio, video, sensor data, and digital fabrication, open the door to a range of new media for people to interact with computers, people, and the world. These new media promise to radically transform creativity by putting the means of design and production in the hands of everyone, and reducing the cost of sharing design knowledge to near zero.

We discussed one specific example, the NeuroMaker, which was realized as an artwork. While the prototype is not necessarily practically useful, the most important finding is that it could be done at all, given a few months, a few thousand dollars, and the right team.

In the rest of this section we consider conclusions and provocative questions raised by this work. First we consider the NeuroMaker prototype itself, and then turn to broader issues.

\subsection{The NeuroMaker - in fact, it could be done}

The NeuroMaker prototype was developed as part of an examination of the future of mass customization and embodied computing, and the implementation demonstrated that these technologies can be integrated using low-cost, widely available technology to create potentially unprecedented tools. Even with limited goals and resources, it was possible to create customized objects that were pleasing to the user co-creators. Our motto was, "It could be done, therefore we had to do it."

Perhaps the most significant contribution has been to provoke thought and raise question about design, creativity, and agency. Some of these questions are discussed here. We need to emphasize that the NeuroMaker was deployed in the context of an art installation; it was not a scientific investigation. Nevertheless, we have made informal observations based on the comments of the patrons at the installation. 


\subsubsection{Mind control? Or placebo effect?}

The NeuroMaker reads the user's EEG, and generates the results from the EEG data. The EEG measurements have only a coarse relation to mental activity, and the parametric equations that generated the decorative patterns were selected to be unpredictable. Significantly, during development and testing, it was difficult to distinguish the results in test mode (i.e., when the input was random numbers) from the actual EEG-generated results. In other words, there was, in fact, no simple or direct relationship between the person's "mental state" and the results produced.

Despite the tenuous connection between the user's thoughts and the output, many people reported that they felt the results were both pleasing and representative of their mood. These reports must surely represent a placebo effect, based on faith in our strong, factual, assertion that the EEG controls the output.

\subsubsection{Collaborative creation}

This work illustrated a case of collaborative design, including the consumer directly in the design and production of decorative objects.

In this case, the object was co-created by:

- An industrial designer, using CAD software tools

- A team of designers, craftspeople, and engineers who assembled the equipment,

- A software engineer, integrating software and data, and

- The consumer, who generated and selected the decorative design.

At a very fundamental level, this collaboration was enabled by the digital representation of the design, which could be manipulated by different people and techniques, then combined into a coherent, viable, manufacturable object.

\subsubsection{Who created the object?}

While the project began out of questions about the role of the designer as mediator, the NeuroMaker actually created a hybrid system in which objects are created through collaboration of the designer and the consumer. This collaborative process led to unexpected questions about agency — who "created" the product?

The NeuroMaker was displayed in the context of a art installation in which users operated the system to create their own cards inscribed with a custom decoration derived from their own brainwaves. We believed that it should have been clear that the objects were made through a collaborative effort of the system designers and the individual user.

Given this, it is interesting to note that many of participants requested that the developers autograph their product, i.e., to ask the designers to assume the role of the "artists" who "created" the work. Despite our emphatic assertions that "you made it yourself", many people nevertheless apparently identified the designers as "authors" of the objects they created, or at least as having had a significant role in the creation.

\subsection{A collaborative context}

This collaboration would not have been possible without an institutional context. Our work in personal fabrication was set in the Champaign Urbana Community Fab Lab, which hosts an eclectic group of designers, artists, engineers, teachers, and others; who 
share a common interest in personal fabrication (Watson, 2011). In this setting, mutual teaching and learning is fostered, and multidisciplinary collaboration is the norm.

Similarly, our work in embodied computing has developed under the umbrella of several virtual institutes at the University of Illinois, Urbana-Champaign (eDream, 2011; I-CHASS, 2009; IACAT, 2011; Illinois Informatics Institute, 2012). These institutions are specifically designed to bring to bear diverse theories and practices, through mutual teaching, learning, and translation across conceptual boundaries. This effort is far more than equipping artists and designers with better tools, for we are also equipping technologists with crucial new insights, techniques, and approaches. Expert performing artists bring important skills and practices, including the ability to perform precise and replicable actions, and rich bodies of theory developed in the study of music, dance, and theatrical performance. Musicians have a deep understanding of the "feature space" of sound, as well as well-developed concepts about the abstract meaning of music and musical performance (Thompson, 2009). Similarly, there is a long history of first-person studies of movement and dance, which provide deep understanding and formal and informal knowledge about embodiment and motion (Schiphorst, 2009a, 2009b; Schiphorst, 2009), and theatrical artists have deep knowledge of interpersonal interactions implemented through story and role playing (Freeman, 2004; Laurel, 1991; McGonigal, 2007, 2011; McGonigal, 2006; Miller, 2004; Salen \& Zimmerman, 2004).

\subsection{Implications}

The technologies discussed here are transforming creativity in different ways, made possible by the power of ubiquitous networked computing. These developments are possible through technologies that cross the boundaries of physical and virtual worlds, through digital data related to the physical world. This crossing is decisive, for, once something is digitized, we can utilize all the capabilities of networked computing - it becomes possible to create, edit, and compute, and also to mix, remix, and share.

Digital data related to the physical world has the advantages of any data: it can be transported and shared at low cost, it can be searched and optimized, and it can be kept for long periods of time without spoiling. Furthermore, the whole is greater than its parts; we can easily combine digital data from any source, to create unprecedented systems.

The NeuroMaker project described here was, in this sense, very "easy" to implement. Given the basic technology which worked with digital representations of the body and physical objects, the overall system could be realized by software and data integration - "just a bit of programming". Thus, these technologies enable us to bring together the right combination of expertise, to build an otherwise "impossible" artifactsuch as the NeuroMaker 1.0.

The development of whole body interfaces and similar environments is expanding how people can interact with information, and also brings the power of information to previously "analog" interactions (England, 2011b). While, it is not yet possible to "sing" a chair into being, or to "dance" together a salad, it definitely is possible to sculpt a lampshade using karate (Fluidforms, 2011) and to steer a computation with a violin (Smith \& Garnett, 2011a). We do not know where this technology will lead, but it should transform the way people interact with computer (England, 2011b), how they interact with each other via computer networks (Smith, 2011), and how people interact with their built environment (Gross \& Green, 2012). 
Personal fabrication is rightly recognized as a revolutionary technology, possibly on a par with the industrial revolution (Anderson, 2010; Gershenfeld, 2005; Mota, 2011; Rischau, 2011). While it is clear that personal fabrication will not soon displace all mass production (Johnson, 2010), it seems certain that the transformations we have seen in the use of information - the realm of "bits" - will now occur in the realm of "atoms" as well. The cultural changes are already underway, as well as the first economic tremors. It isn't yet possible for your closet to fabricate a new wardrobe for you each day, but you definitely can download and "print" a new coffee cup every morning (e.g., dozens of designs that can be discovered by a search at (Makerbot Industries, 2011b))

We cannot know where this will lead, but we believe it will be profound. Just as digital music and video have become universally available, nearly for free, and can be uploaded as well as consumed, the ability to make things will be universally shared. In this, we are surely putting tools in the hands of the workers.

Ultimately, we believe these technologies will have profound psychological and cultural effects. Embodied computing humanizes interactions with digital systems, and most likely will create healthier and more enjoyable work experiences (England, 2011b). Personal fabrication democratizes the creation of objects, with both economic benefits and a concomitant feeling of personal agency (Gershenfeld, 2005; Moss, 2011; Watson, 2011).

In many ways, the cultural effects are the most exciting. It is thrilling when a kid's face light up and he or she says, possibly for the first time, "I made this". And that is only the beginning - they inevitably teach other kids.

\section{Acknowledgements}

Many Thanks for their large or small contributions to this project: Gary Watson, Mary $\mathrm{K}$ Watson, Luke Scharf, Betty Barrett, Guy Christopher, Anna Gutsch, Huang Li, Yi Lao.

Thanks to the support and encouragement from countless individuals at the National Center for Supercomputing Applications (NCSA) and the Institute for Computing in Humanities, Arts, and Social Science" (I-CHASS) at the University of Illinois, Urbana Champaign.

The Project would not have been possible without the support and funding of the Champaign Urbana Community Fab Lab.

Robert McGrath was partly funded through by the National Science Foundation, ARC1025298 “Augmented Reality for Understanding Social and Environmental Science”.

\section{References}

Anderson, C. (2010, February). In the next industrial revolution, atoms are the new bits. Wired, 18, 58-67.

ArtSoft. (2011). The home of Mach3 and LazyCam. Retrieved from http://www.machsupport.com/.

Billinghurst, M., Kato, H., \& Poupyrev, I. (2001). The MagicBook - Moving seamlessly between reality and virtuality. IEEE Computer Graphics and Applications, 21(3), 6-8.

Buildyourcnc. (2011). Desktop CNC machine kit - bluechick. Retrieved from http://buildyourcnc.com/DesktopCNCMachineKitblueChick.aspx.

Castranova, E. (2007). Exodus to the virtual world: How online fun is changing reality. New York: Palgrave Macmillen. 
Eck, D. J. (2001). Java components for mathematics. Retrieved from http://math.hws.edu/javamath/.

eDream. (2011). eDream: Emerging digital research and education in arts media institute. Retrieved from http://edream.illinois.edu/.

England, D. (2011a). Whole body interaction: An introduction. In D. England (Ed.), Whole Body Interaction (pp. 1-5). London: Springer.

England, D. (Ed.). (2011b). Whole body interaction. London: Springer.

Fluidforms. (2011). CASSIUS Punching bag design interface. Retrieved from http://vimeo.com/2847325.

Freeman, D. (2004). Creating emotion in games. Berkeley: New Riders.

Gershenfeld, N. (2005). Fab: The coming revolution on your desktop-From personal computing to personal fabrication. New York: Basic Books.

Gross, M. D., \& Green, K. E. (2012). Architectural robotics, inevitably. Interactions, 19(1), 28-33. DOI: 10.1145/2065327.2065335

Haacke, E. M., Brown, R. F., Thompson, M., \& Venkatesan, R. (Eds.). (1999). Magnetic resonance imaging: Physical principles and sequence design. New York: J. Wiley \& Sons.

Hornecker, E. (2011). The role of physicality in tangible and embodied interactions. Interactions, 18(2), 19-23. DOI: 10.1145/1925820.1925826

I-CHASS. (2009). Institute for computing in humanities, arts, and social science. Retrieved from http://www.chass.illinois.edu/Index/Index.html.

IACAT. (2011). The institute for advanced computing applications and technologies. Retrieved from http://iacat.illinois.edu/.

Illinois Informatics Institute. (2012). Illinois informatics institute: Invent, imagine, inspire. Retrieved from https:// www.informatics.illinois.edu/icubed/.

Isbister, K. (2011). Emotion and motion: Games as inspiration for shaping the future of interface. Interactions, 18(5), 24-27. DOI: 10.1145/2008176.2008184

Ishii, H., \& Ullmer, B. (1997). Tangible bits: Towards seamless interfaces between people, bits and atoms. Paper presented at the CHI, Atlanta.

Johnson, J. (2010, Jan 26). Atoms are not bits; Wired is not a ausiness magazine. Retrieved from http://gizmodo.com/5457461/atoms-are-not-bits-wired-is-not-abusiness-magazine.

Json.org. (2011). Introducing Json. Retrieved from http://www.json.org/.

Krueger, M. W. (1991). Artificial reality II. Reading, Mass.: Addison Wesley.

Latulipe, C., Wilson, D., Huskey, S., Word, M., Carroll, A., Carroll, E., . . . Lottridge, D. (2010). Exploring the design space in technology-augmented dance. Paper presented at the Proceedings of the 28th of the international conference extended abstracts on Human factors in computing systems, Atlanta, Georgia, USA.

Laurel, B. (1991). Computers as theatre. Reading, MA: Addison Wesley.

LilyPond. (2011). LilyPond. Retrieved from http://lilypond.media.mit.edu/.

Make. (2011). Make: Technology on your time. Retrieved from http://makezine.com/.

Makerbot Industries. (2011a). MakerBot. Retrieved from http://www.makerbot.com/.

Makerbot Industries. (2011b). Thingiverse. Retrieved from http://www.thingiverse.com/.

McFarland, D. J., \& Wolpaw, J. R. (2011). Brain-computer interfaces for communication and control. Commun. ACM, 54(5), 60-66. DOI: 10.1145/1941487.1941506

McGonigal, J. (2007). The puppet master problem: Design for real-world, mission based gaming. In P. Harrigan \& N. Wardrip-Fruin (Eds.), Second Person: Role-Playing and Story in Games and Playable Media. Cambridge: MIT Press.

McGonigal, J. (2011). Reality is broken: Why games make us better and how they can change the world. New York: Penguin Press.

McGonigal, J. E. (2006). This might be a game: Ubiquitous play and performance at the 
turn of the twenty-first century. (Doctor of Philosophy Dissertation), University of California, Berkeley, Berkeley. Retrieved from http:// antiboredom.org/uploads/McGonigal_THIS_MIGHT_BE_A_GAME_sm.pdf.

McGrath, R. E., Craig, A., Bock, D., \& Rocha, R. (2011). Augmented reality for an ethnobotany workbook: Institute for computing in the humanities, social sciences and arts (I-CHASS), University of Illinois, Urbana-Champaign.

McGrath, R. E., Rischau, J., \& Craig, A. B. (2011). Personalized manufacturing meets embodied computing. Paper presented at the Workshop on Semi-Automated Creativity at ACM Creativity and Computation, Atlanta. Retrieved from http://computationalcreativity.org/sac/wpcontent/uploads/2011/10/mcgrath_etal_sac2011.pdf.

Miller, C. H. (2004). Digital storytelling: A creator's guide to interactive entertainment. Burlington, MA: Elsevier.

Mistry, P., Maes, P., \& Chang, L. (2009). WUW - wear Ur world: A wearable gestural interface. Paper presented at the Proceedings of the 27th international conference extended abstracts on Human factors in computing systems, Boston, MA, USA.

Moss, F. (2011). The sorcerers and their apprentices. New York: Crown Business.

Mota, C. (2011). The rise of personal fabrication. Paper presented at the Creativity \& Cognition, Atlanta.

NCSA. (2011). The NeuroMaker: When science meets design. Retrieved from http://www.ncsa.illinois.edu/News/Video/2011/neuromaker.html.

NeuroSky. (2010a). MindSet communications protocol. Retrieved from http://developer.neurosky.com/docs/doku.php?id=thinkgear_communications_protoc ol.

NeuroSky. (2010b). ThinkGear socket protocol. Retrieved from http://developer.neurosky.com/docs/lib/exe/fetch.php?media=app_notes:thinkgear_so cket_protocol.pdf.

NeuroSky. (2011). NeuroSky: Brain wave sensors for every body. Retrieved from http://www.neurosky.com/.

New Media Collective. (2011). The pool. Retrieved from http://pool.newmedia.umaine.edu/.

Niedermeyer, E., \& da Silva, F. L. (Eds.). (1982). Electroencephalography, basic principles, clinical applications, and related fields. Baltimore: Urban \& Schwarzenberg.

Nintendo. (2010). Wii.com. Retrieved from http://wii.com/.

Norton, D., Heath, D., \& Ventura, D. (2011). A collaboration with DARCI. Paper presented at the Semi-Automated Creativity Workshop, Atlanta. Retrieved from http://computationalcreativity.org/sac/wpcontent/uploads/2011/10/norton.heath.ventura.pdf.

Openhardwaresummit.org. (2011). Open hardware summit. Retrieved from http://www.openhardwaresummit.org/.

Pentland, A. (2008). Honest signals: How they shape our world. Cambridge, Mass.: MIT Press.

Pietrowicz, M., McGrath, R. E., Garnett, G., \& Toenjes, J. (2010). Multimodal gestural interaction in performance. Paper presented at the Whole Body Interfaces Workshop at $\quad$ CHI 2010, Atlanta. Retrieved from http://lister.cms.livjm.ac.uk/homepage/staff/cmsdengl/WBI2010/documents2010/Pietr owicz.pdf.

Pine, B. J. (1993). Mass cusomization: The new frontier in business competition. Boston: Harvard Business School Press.

Rischau, J. (2011). Custom digital fabrication in industrial design. (MFA), University of Illinois, Urbana-Champaign, Urbana. Retrieved from 
http://hdl.handle.net/2142/26045.

Salen, K., \& Zimmerman, E. (2004). Rules of play: Game design fundamentals. Cambridge: The MIT Press.

Schiphorst, T. (2009a). Body matters: The palpability of invisible computing. Leonardo, 42(3), 225-230.

Schiphorst, T. (2009b). Soft(n): Toward a somaesthetics of touch. Paper presented at the Proceedings of the 27th international conference extended abstracts on Human factors in computing systems, Boston, MA, USA.

Schiphorst, T. H. H. M. (2009). The varieties of user experience: Bridging embodied methodologies from somatics and performance to human computer interaction (Doctoral Dissertation). University of Plymouth, Plymouth.

Shapeways.com. (2011). Shapeways. Retrieved from http://www.shapeways.com/about/.

Sherman, W. R., \& Craig, A. B. (2003). Understanding virtual reality: Interface application and design. San Francisco: Morgan Kaufmann.

Smid, P. (2008). CNC programming handbook (3rd ed.). New York: Industrial Press.

Smith, B. D. (2011). Telematic composition. (Ph.D. Dissertation), University of Illinois, Urbana-Champaign, Urbana. Retrieved from http://hdl.handle.net/2142/29843.

Smith, B. D., \& Garnett, G. E. (2011a). High performance computing for music. Paper presented at the International Computer Music Conference, Huddersfield, UK.

Smith, B. D., \& Garnett, G. E. (2011b). The self-supervising machine. Paper presented at the New Interfaces for Musical Expression (NIME), Oslo. Retrieved from http://www.nime2011.org/proceedings/papers/B21-Smith.pdf.

Swartza, B. E., \& Goldensohnb, E. S. (1998). Timeline of the history of EEG and associated fields. Electroencephalography and Clinical Neurophysiology, 106(2), 173-176. DOI: 10.1016/s0013-4694(97)00121-1

Thompson, W. F. (2009). Music, thought, and feeling: Understanding the psychology of music. Oxford: Oxford University Press.

Vallabhaneni, A., Wang, T., \& He, B. (2005). Brain-Computer interface. In B. He (Ed.), Neural Engineering (pp. 85-121): Springer US.

Van Laerhoven, K., \& Cakmacki, O. (2000, October 16-17). What shall we teach our pants? Paper presented at the Fourth International Symposium on Wearable Computers, Atlanta.

Wang, P. S.-P. (Ed.). (2011). Pattern recognition, machine intelligence and biometrics. Beijing: Higher Education Press.

Watson, G. (2011). The Champaign-Urbana Community Fab Lab. Interactions, 18(5), 86-87.

Wikipedia. (2011a). Electroencephalography. Retrieved from http://en.wikipedia.org/wiki/Electroencephalography.

Wikipedia. (2011b). G-code. Retrieved from http://en.wikipedia.org/wiki/G-code.

Wikipedia. (2011c). Magnetic resonance imaging. Retrieved from http://en.wikipedia.org/wiki/Magnetic resonance_imaging.

Wikipedia. (2011d). Wii remote. Retrieved from http://en.wikipedia.org/wiki/Wii_Remote.

Williams, D., Ducheneaut, N., Xiong, L., Zhang, Y., Yee, N., \& Nickell, E. (2006). From tree house to barracks: The social life of guilds in World of Warcraft. Games and Culture, 1(4), 338-361..

Wohlsen, M. (2011). Biopunk: DIY scientists hack the software of life. New York: Penguin.

Xiu, Y., Wan, Z.-K., \& Cao, W. (2011). A constructive approach toward a parametric patter-making model. Textile Research Journal, 81(10), 979-991. 